\title{
Supramolecular Assemblies of Cucurbiturils with Photoactive, $\pi$-conjugated Chromophores
}

\author{
Ahmet $\operatorname{Koc}^{[\mathrm{a}]}$ and Dönüs Tuncel*a, b]
}

\begin{abstract}
Supramolecular assemblies of cucurbituril (CBn) homologues with $\pi$-conjugated chromophores will be overviewed. Special emphasis will be given to the effect of $\mathrm{CBn}$ on the optical properties of conjugated oligomers and
\end{abstract}

polymers. How supramolecular complexes of $\pi$-conjugated chromophores including porphyrin derivatives, conjugated oligomers and polymers with $\mathrm{CBn}$ could be utilized in the theranostic and photonic applications will also be discussed.

Keywords: Cucurbiturils · supramolecular assemblies $\cdot \pi$-conjugated chromophores $\cdot$ porphyrins $\cdot$ conjugated polymers

\section{Introduction}

In this review, we are going to discuss the supramolecular assemblies of cucurbituril ( $\mathrm{CBn})$ homologues with photoactive, conjugated chromophores. Photoactive, $\pi$-conjugated chromophores have many important applications in the areas of sensing, catalysis, lasers, imaging, theranostics and photonics. ${ }^{[1-6]}$ However, there are some drawbacks that should be overcome in order to utilize them in a wider context including stability (chemical, thermal, photo), solubility, poor optical performance (low quantum yields, low lifetime) due to aggregation.

Here we will present recent literature examples that $\mathrm{CB}$ homologues might offer some solutions to tackle these problems. We will first start with assemblies of CBs with small photoactive dyes and present examples on their use in emerging applications and then we will deal with supramolecular structures based on the interactions of CBs with porphyrin derivatives, conjugated oligomers and polymers.

We will especially emphasize the application of these nanostructures in the areas of theranostics and photonics.

\subsection{Cucurbit[n]uril Homologues}

Cucurbit[n]uril $(\mathrm{CBn})$ has a robust macrocyclic structure and is synthesized by the acid-catalyzed condensation reaction between glycoluril and formaldehyde. Although the first synthesis of CB dates back to Behrend's work in 1905, its structure has not been clarified until 1980s. ${ }^{[7]} \mathrm{CB}$ chemistry, that have been silent for many decades, was stimulated in 1981 by the study of Mock et al. elucidating the unique pumpkin-shaped macrocyclic hexameric structure of cucurbit [6] uril (CB6), which composes two carbonyl-decorated hydrophilic portals and a hydrophobic cavity. ${ }^{[8]}$ In the very beginning of our millennium, independent works of Kim et al. and Day et al. marked the discovery of new CB homologues, which are $\mathrm{CB} 5, \mathrm{CB} 7, \mathrm{CB} 8$ and $\mathrm{CB} 10$, containing 5, 7, 8 and
10 glycoluril units, respectively. ${ }^{[9]}$ The new members of $\mathrm{CB}$ family that shows remarkable differences in their cavity sizes, guest binding affinities, size selectivity and water-solubility attracted huge interest by the supramolecular chemists. ${ }^{[10]}$ Among them, CB7, with good water solubility, appropriate guest size selectivity and binding affinity, have been the most in demand up to now.

Compared to other well-known macrocyclic host molecules such as cyclodextrins, calixarenes and crown ethers, CBs not only exhibit the similar low-toxic nature, but they also offer much higher chemical stability and far better guest binding affinity and size selectivity. ${ }^{[10,11]}$ Cooperative impact of both ion-dipole interactions at the carbonyl portals and hydrophobic interaction inside the cavity provides excellent host-guest complexation with a myriad of neutral hydrophobic or cationic species. ${ }^{[10]}$ The outstanding features of $\mathrm{CB}$ homologues as host molecules originate from their unique structure. The narrow carbonyl portals at both upper and lower parts of the molecule and wider hydrophobic cavity in the middle is believed to be two of the main features for the superior guest binding affinities. Additionally, these structural properties of $\mathrm{CBs}$ enabled chemists to construct large supramolecular architectures like molecular machines, rotaxanes and polyrotaxanes. ${ }^{[11,12]}$

\subsection{Assembly of Cucurbiturils with Small Photoactive Dyes}

The interaction of small fluorescent dyes with CBs and the effect of $\mathrm{CBs}$ on their photochemical and photophysical

[a] A. Koc, D. Tuncel

Department of Chemistry, Bilkent University, Ankara 06800, Turkey

[b] D. Tuncel

UNAM-National Nanotechnology Research Center, Institute of Materials Science and Nanotechnology, Bilkent University, Ankara 06800, Turkey

E-mail: dtuncel@ fen.bilkent.edu.tr 
properties have been extensively studied especially by Nau and co-workers and there are also very insightful review papers available on this topic. ${ }^{[13-16]}$ Therefore, we will not go into details of the works covered in those papers but present the recent works on the assembly of fluorescent dyes and their applications which were not covered in those reviews.

Cucurbit[7]uril (CB7) among other CB homologues has mostly involved in these studies due to its appreciable amount of solubility in aqueous media. The effect of $\mathrm{CB} 7$ on the optical properties of the following dyes (Figure 1) have been investigated: ${ }^{[13-16]}$ Macrolex Yellow 10 GN, Dapoxyl, and 4(dimethylamino) benzonitrile), rhodamine 6G, rhodamine 123 , tetramethylrhodamine, cresyl violet, fluorescein, coumarin 102, pyronin B, pyronin $\mathrm{Y}$, two cyanine 5 and one cyanine 3 derivative, and IR140, IR144 as well as coumarin-derivative dyes, ${ }^{[7]}$ 7-(diethylamino)-N-(1,3-dihydroxy-2-(hydroxymethyl) propan-2-yl)-2-oxo-2H-chromene-3-carboxamide and $\mathrm{N}-(1,3-$ dihydroxy-2-(hydroxymethyl)propan-2-yl)-11-oxo-

2,3,5,6,7,11-hexahydro-1H-pyrano[2,3f]pyrido[3, 2,1-ij]

quinoline-10-carboxamide.

It was shown that the presence of CB7 affects significantly the optical properties of these aforementioned dyes. Upon encapsulation of dyes by CB7s, a spectral shift is observed as well as an increase in their fluorescence quantum yields, enhanced brightness and photostability. Moreover, fluorescence lifetimes of the encapsulated dyes are prolonged due to the low polarizability of the interior of the macrocycle. The high affinity of the carbonyl decorated portals of CBs towards cations results in a considerable shift of protonation equilibria and assists the protonation of fluorescent dyes. ${ }^{[17,18,23,24]}$

The effect of CB7 on the lasing characteristics of rhodamine B and sulforhodamine B (Kiton Red S) dyes in aqueous solution were investigated in a narrow-band dye laser setup. It was found that the photostability and thermo-optical properties of dyes upon complexation with CB7 in aqueous solutions improved significantly as compared to the uncomplexed dyes in ethanol solution. The experimental results showed that aqueous solutions of the supramolecular complexes of dyes with CB7 are highly appealing active medium for high repetition rate dye laser operation. ${ }^{[19]}$

It was also demonstrated that excimer emission can be observed when $p$-dimethylaminobenzonitrile (DMABN) is encapsulated by CB8 forming a ternary complex (CB8:2DMABN). Heating of the CB8.2DMABN complex from 0 to $100{ }^{\circ} \mathrm{C}$ results in the dissociation of the ternary complex and restoration of the dual-emission properties of the monomer. However, the cavity of CB7 is found to be not large
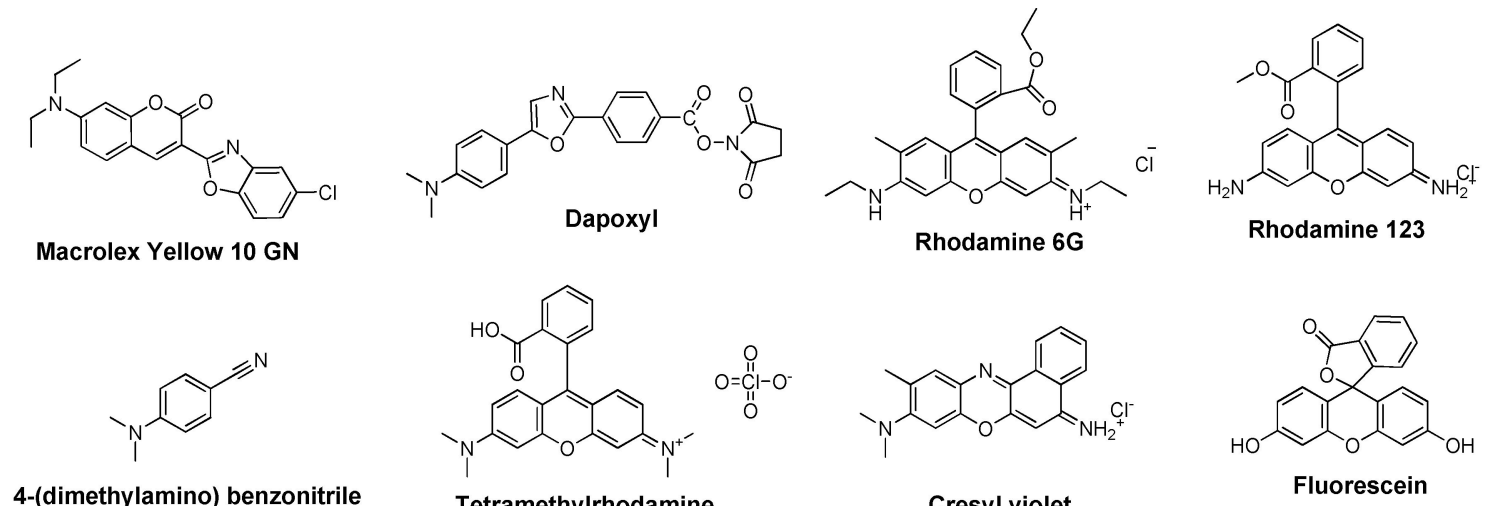

4-(dimethylamino) benzonitrile

TetramethyIrhodamine

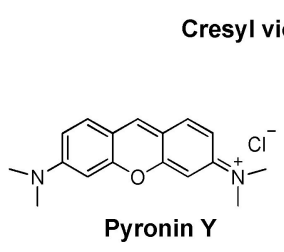

Pyronin B

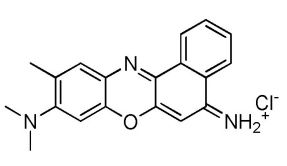

resyl violet
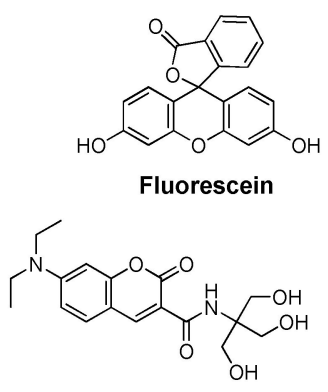

7-(diethylamino)- $N$-(1,3-dihydroxy-2(hydroxymethyl)propan-2-yl)-2-oxo-2Hchromene-3-carboxamide (AGD)

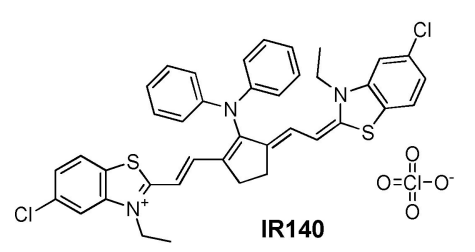

IR140

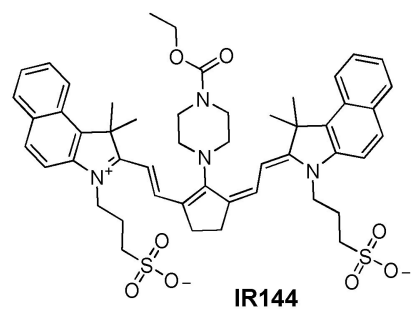

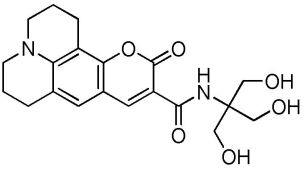

$N$-(1,3-dihydroxy-2-(hydroxymethyl)propan-2yl)-11-oxo-2,3,5,6,7,11-hexahydro-1Hpyrano[2,3-f]pyrido[3,2,1-ij]quinoline-10carboxamide

Figure 1. Molecular structures of photoactive dyes. 
enough to accommodate two DMABN molecules. Ternary complex formation could also be prevented by introducing ethyl instead of methyl groups at the amino terminus of the aminobenzonitrile guest as in this case two DMABN molecules could not fit the cavity of CB8. ${ }^{[2]}$

One of the most important effects of CBs on the photoactive chromophores is an increase in their fluorescence quantum yields. One of the reasons of emission quenching is due to aggregation formation through inter or intramolecular interactions among the chromophores as $\pi-\pi$ stacking, $\mathrm{H}$ bonding, etc. If these interactions are minimized or prevented, quenching can be avoided. Aggregate formation can be prevented by encapsulating the chromophores with suitablysized macrocycles. CB homologues can serve well to this purpose. CB7 is highly desirable for its good water solubility and relatively large cavity but CB8 might be a better option for larger chromophores that cannot fit into the cavity of CB7. Although CB8 has a poor solubility in water that can be improved upon inclusion complex formation and as well as with addition of aqueous salt or acid solutions.

Perylene bis(diimide) (PDI) dyes are highly prone to aggregate formation due to their large hydrophobic aromatic part which can interact through $\pi-\pi$ stacking. Aggregate formation quenches its emission as well as decreases the solubility especially in aqueous media. It was shown that this aggregate caused emission quenching can be prevented by encapsulating the PDI dye with CB8. ${ }^{[20]}$ The encapsulation enhances its photochemical and chemical stability. These inclusion complexes exhibit redox stimuli-responsive behavior to the competitive guests and can be switched between the non-fluorescent and fluorescent states by reduction and oxidation. Interestingly, although PDI is already a large guest, CB8 could accommodate suitable dicationic or electron-rich second guests by forming ternary complexes (Scheme 1). Authors anticipate that this feature can be used for the reversible formation of higher-order supramolecular architectures in water. ${ }^{[21]}$
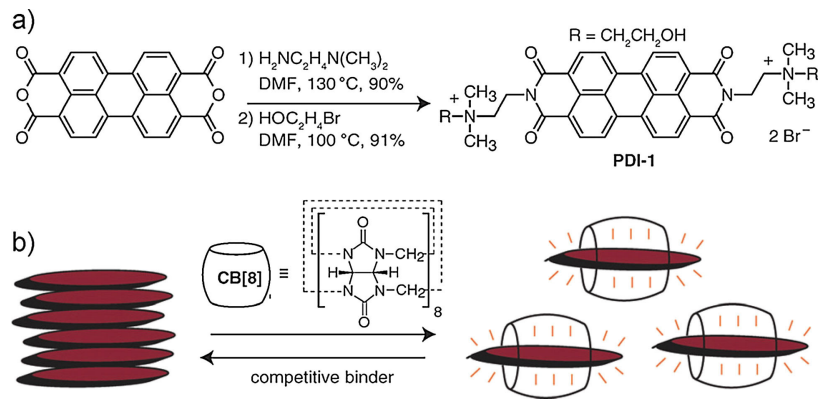

Scheme 1. a) Synthesis of PDI-1. b) Reversible deaggregation of PDI1 stacks in water upon addition of CB8. (Reprinted with permission from ref. 21. Copyright 2012 Wiley-VCH Verlag $\mathrm{GmbH} \&$ Co. KGaA.)

Disaggregation ability of CBs was also exploited in the use of photostable fluorescent label for imaging mitochondria in living cells. For this purpose, a near infrared (NIR) dye, 4-1pyrenyl-vinyl-N-ethylquinolinium chloride was synthesized (Scheme 2) by introducing a hydrophilic cation moiety $\mathrm{N}$ ethylquinolinium which formed an inclusion complex with CB8 in aqueous solution which disrupted the aggregation of the dye, leading to a 30 -fold fluorescence increase. ${ }^{[22]}$

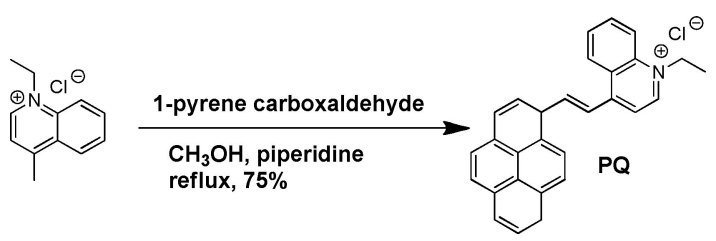

Scheme 2. Synthesis of a near infrared (NIR) dye, 4-1-pyrenyl-vinyl$\mathrm{N}$-ethylquinolinium chloride. ${ }^{[21]}$

Host-assisted dye protonation is one of the interesting features of the CBs. ${ }^{[17,18,23,24]}$

For instance, a fluorescent dye based on 3-amino-9ethylcarbazole with a diamino-alkyl anchor was reported which displays a large and predictable dual fluorescence response (100-fold increase at $375 \mathrm{~nm}$ and 9-fold decrease at $458 \mathrm{~nm}$ ) upon encapsulation by CB and a strong affinity for cation receptor macrocycles, in particular CB6. This characteristic was used as CB-responsive fluorescent switch operating through a pKa shift (Figure 2) and the enzymatic activity of lysine decarboxylase was monitored by taking advantage of this switch. ${ }^{[17]}$

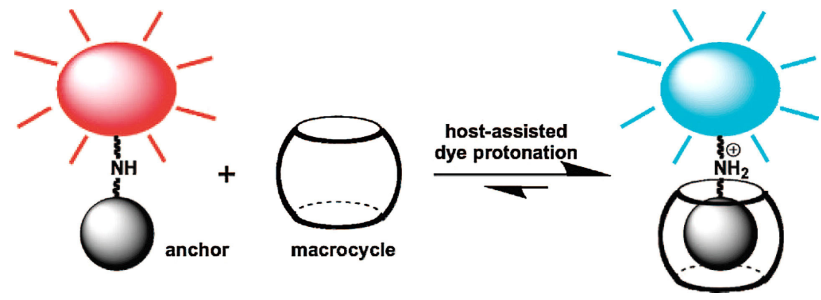

Figure 2. Schematic presentation of CB-responsive fluorescent switch operating through a pKa shift. ${ }^{16}$ (Reprinted with permission from ref. 17. Copyright 2008 American Chemical Society.)

Very recently, ternary complex formation ability of CB 8 was used in the design of activatable supramolecular photosensitizer (aPS) for targeted cancer imaging and photodynamic therapy (Figure 3). ${ }^{[25]}$ This system is based on the host-guest interaction between biotinylated toluidine blue (TB-B) and CB8 to form 2TB-B@CB8. Moreover, a facile strategy to turn off /on the fluorescence and photodynamic activity of TBB is developed through the reversible assembly/disassembly of 2TB-B@CB[8]. This established system can achieve selective accumulation in tumor, light-up cancer imaging, and enhanced anticancer behavior. Therefore, this work provides a 


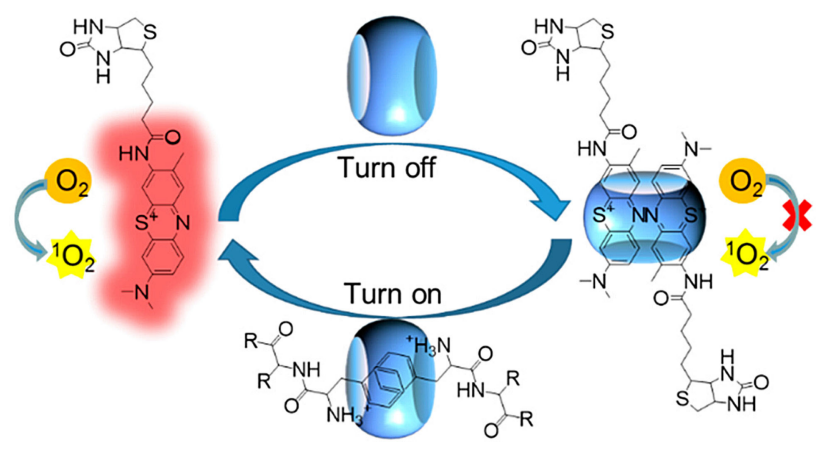

Figure 3. Working principle of CB8-regulated activatable supramolecular photosensitizer for targeted cancer imaging and photodynamic therapy. (Reprinted with permission from ref. 25. Copyright 2016 American Chemical Society.)

novel and promising strategy for the aPS build via simple and facile regulation of supramolecular chemistry. ${ }^{[25]}$

\subsection{Assembly of Cucurbiturils with Porphyrins}

Porphyrins are very interesting multifaceted chromophores exhibiting, photo, redox, electrochemical properties. Due to these properties, they have numerous potential applications including photocatalytic catalysis, light harvesting, sensing, photodynamic therapies. ${ }^{[26]}$ Especially water-soluble derivatives are highly sought after for biological applications. However, due to their large hydrophobic $\pi$-conjugated structures they have tendency to form aggregates in aqueous media and solid state. Therefore, it would be very helpful to find ways to prevent aggregation and render them water soluble. Here again, the macrocycle $\mathrm{CB}$ can lend some solutions to this problem. Accordingly, in this section, we are going to discuss the effect of $\mathrm{CBs}$ on the properties of porphyrin derivatives. ${ }^{[16,27]}$ Figure 4 shows most of the molecular structures of the porphyrin-based compounds discussed in this section.

One of the first studies on CB-porphyrin assemblies reported by our group around 2006. ${ }^{[28]}$ In this work we have synthesized water soluble [5]rotaxane and [5]pseudorotaxane based on cucurbit[6]uril (Figure 4) and anchored to a mesotetraphenyl porphyrin and studied their $\mathrm{pH}$-triggered switching properties. However, we have not study the optical properties of these rotaxanes and pseudorotaxanes. ${ }^{[28]}$

This work was followed by a paper of Isaacs and coworkers who reported the formation and characterization of a series of CB10.porphyrin complexes and the ability of $\mathrm{CB} 10 \cdot 2$ to form ternary complexes with a variety of aromatic amines in water. ${ }^{[2]}$ They have shown that the cavity of the CB10 is large enough to encapsulate the free base and metalated 5,10,15,20-tetrakis(4-N-methylpyridyl)porphyrin (TMPyP) (Figure 4). It was observed that although CB10 underwent an ellipsoidal deformation upon encapsulating the porphyrins, the characteristic optical and electrochemical properties of the porphyrins have been retained. Although these encapsulated porphyrins could be utilized in many important applications, their applications have not been explored so far. ${ }^{[29]}$

Around the same time, the non-covalent interaction of TMPyP with CB7 in water was studied. It was shown that CB7s encapsulate methylpyridyl part of the porphyrin to form supramolecular assembly with high binding constant. ${ }^{[30]}$ Optical properties of the assembly of TMPyP with CB7 were investigated through steady-state and time-resolved fluorescence spectroscopy. The presence of CB7 caused considerable changes in the emission spectra of the TMPyP,

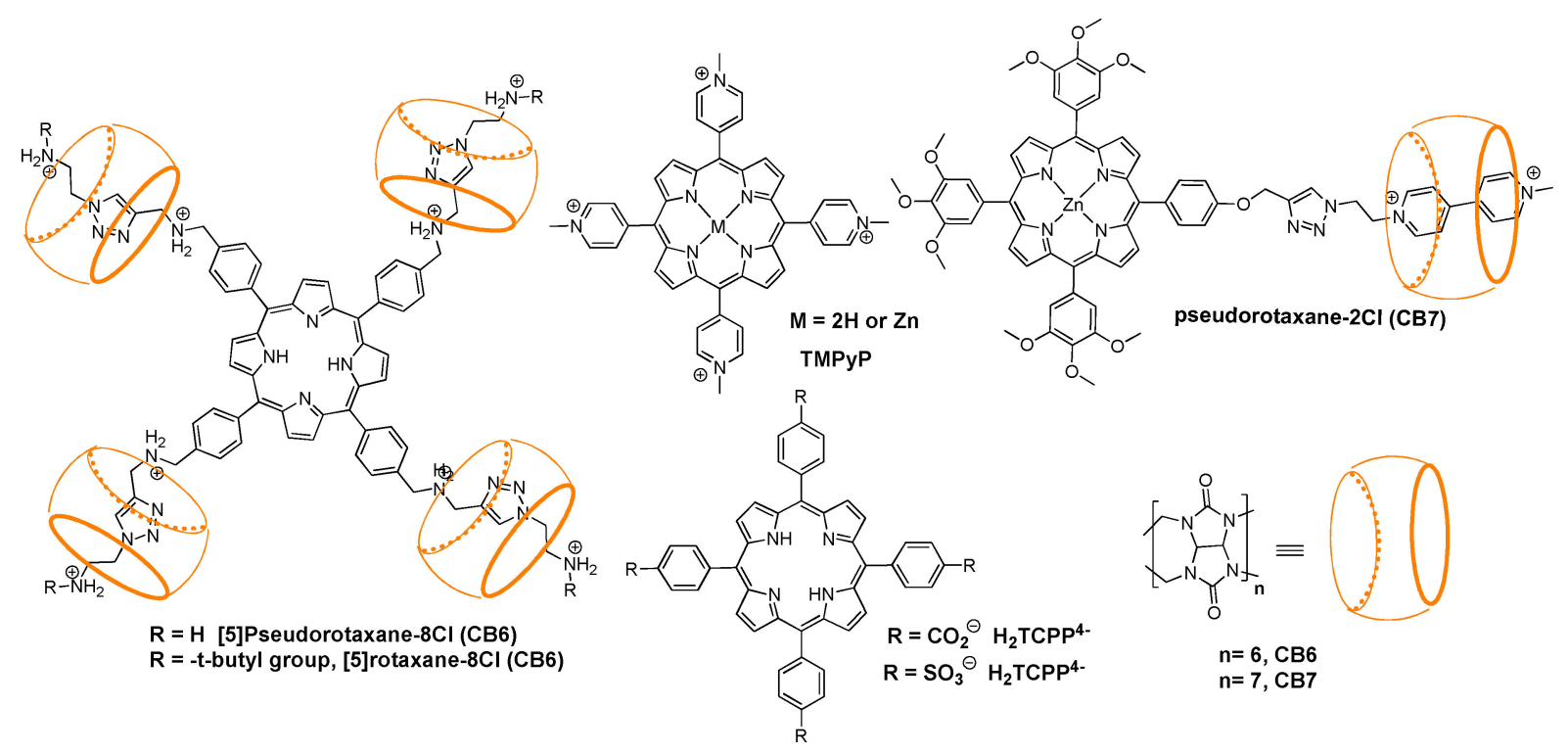

Figure 4. Molecular structures of the porphyrin-based compounds discussed in this section. 
broad fluorescence spectrum of TMPyP appeared as two narrow bands centered at 655 and $717 \mathrm{~nm}$ as well as an incerase in the fluorescence quantum yield and fluorescence time were noted. ${ }^{[30]}$ By taking advantage of the ability of CB7 forming supramolecular assembly with TMPyP, the CB7AgNP conjugates were used for the uptake and release of TMPyP. ${ }^{[31]}$

Jiang and coworkers have reported the synthesis and characterization of porphyrin and tetramethylated CB6(TMeQ[6]) based porous materials and the iodine adsorption capacity of these materials. ${ }^{[32]}$ These porous materials were assembled through host-guest complexation of cationic tetrakis(4-pyridyl)porphyrin species of $(\mathrm{H} 6 \mathrm{TPyP})^{4} \cdot 4 \mathrm{Cl}^{-}$and $\mathrm{TMeQ}[6]$. The structure of the complex was confirmed by single crystal $\mathrm{x}$-ray diffraction analysis. ${ }^{[32]}$

The complexes of alkaline or alkaline earth metal cations, such as $\mathrm{K}^{+}$and $\mathrm{Ca}^{2+}$ with $\mathrm{CB} 7$ or $\mathrm{CB} 8$ were treated with anionic porphyrins, meso-tetra(4-carboxyphenyl)-porphyrin (H2TCPP4) and meso-tetra(4-sulfonatophenyl)-porphyrin (H2TPPS4) in aqueous media to form coordination, network type structures which probably folds into particles or remain as flakes. The sizes of particles are determined as in the range of $0.1-1.0 \mu \mathrm{m}$ by SEM and AFM and depend on the types of CB (CB7 or CB8), cations used and their concentrations. Although a cellular uptake study was conducted in A549 cell lines and the nanoparticle incubated cells were imaged confocal laser scanning microscopy (CLSM), there is not much details provided on the cytotoxicity. ${ }^{[33]}$

Porphyrin dyads were prepared through a click reaction between alkyne Zn-porphyrin and azido-functionalized viologens, in which $\mathrm{Zn}$-porphyrin acts as a donor and viologen as an acceptor. When these dyads are treated with CB7, CB7 could thread the viologen unit to form a pseudorotaxane which allows the modulation of electron transfer from the $\mathrm{Zn}$ porphyrin to the viologen. Its structure is shown in Figure 4. Moreover, pseudorotaxane formation caused an enhancement in the fluorescence quantum yield of the porphyrin and a significant shift in the reduction potentials of the viologen moiety. ${ }^{[34]}$

One of the most important properties of porphyrin is the ability to generate reactive oxygen species that can be used in photodynamic therapy. Recent designs involve the use of porphyrin-CB supramolecular assemblies as photosensitizers to regulate the photophysical properties of porphyrins in the presence of CBs (Figure 5). For example, naphthalenemethylpyridinium attached porphyrin (TPOR) was allowed to form inclusion complex with CB7 in water to be utilized as a photosensitizer for antibacterial treatment. ${ }^{[35]}$ Although in the presence or absence of CB7, TPOR molecules undergo selfassembly to form nanostructures in water, the size and the nature of the nanostructures differ. An increase of the fluorescence intensity of porphyrins is observed upon the binding of CB7 to TPOR as the self-quenching of the excited states are supressed. Moreover, singlet oxygen generation efficiency of TPOR enhanced in the presence of CB7. The antibacterial activities of TPOR and TPOR/CB7 toward
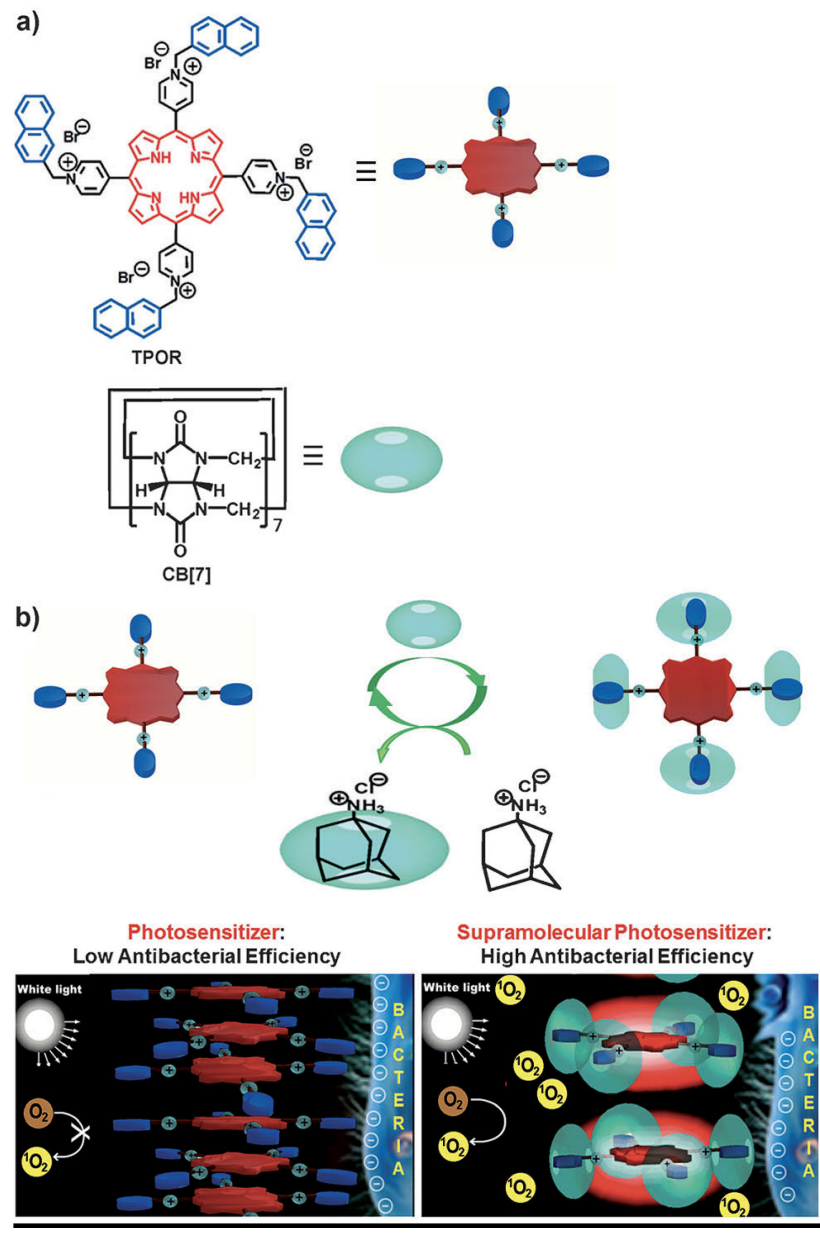

Figure 5. a) Chemical structures of the TPOR (photosensitizers) and CB7. b) The construction of TPOR/(CB7) $)_{4}$ supramolecular photosensitizers and the mechanism for the enhanced antibacterial efficiency of TPOR/(CB7) $)_{4}$ compared with that of TPOR. (Reprinted with permission from ref. 35. Copyright 2013 Wiley-VCH Verlag $\mathrm{GmbH} \&$ Co. KGaA.)

Escherichia coli (E. coli), a Gram-negative bacteria were investigated and TPOR/CB7 was found be very efficient photosensitizer for antibacterial photodynamic therapy. ${ }^{[35,36]}$

Wang and coworkers demonstrated the ability of ternary complex of CB8 with a photosensitizer and the residue of a protein in oxidative photo cleavage of proteins. ${ }^{[37]}$ An inclusion complex of CB8 with a photosensitizer for photodynamic therapy, 5,10,15,20-tetrakis(1-methyl-4-pyridinio)porphyrin tetra(p-toluenesulfonate) (TMPyP, was found to be binding to bovine serum albumin (BSA) very efficiently. Ternary complex of CB8 with TMPyP and the tryptophan part of the BSA place the photosensitizer in a close proximity to protein. This is important as upon light irradiation generated singlet oxygens will be kept and available for damaging the proteins that can regulate the cell signaling process and apoptosis of cells. ${ }^{[37]}$ 
Fuenteal and coworkers have extended the previous works on the ternary complexes of CBs with proteins and photosensitizers to investigate the details and shed light on how to improve the photoactivity of photosensitizers used in the photodynamic therapy of cancer. ${ }^{[38]}$ Acridine orange $\left(\mathrm{AO}^{+}\right)$ was selected as a model photosensitizer and compared it with methylene blue $\left(\mathrm{MB}^{+}\right)$and a cationic porphyrin $\left(\mathrm{TMPyP}^{4+}\right)$. They noted that the photoactivity of photosensitizers were altered upon inclusion complex formation with CBs. While the photooxidation of HSA was enhanced in the presence of the inclusion complex of $\mathrm{AO}^{+}$with $\mathrm{CB} 7$, it was decreased in the presence of inclusion complex of CB8 with $\mathrm{AO}^{+}$. Consequently, reactive oxygen species generation efficiency and protein fragmentation were also increased. The triplet excited state lifetimes of all the photosensitizers were lengthened by their encapsulation by $\mathrm{CB} 7$ or $\mathrm{CB} 8$, while the singlet oxygen quantum yield was enhanced only for $\mathrm{AO}^{+}$and $\mathrm{TMPyP}^{4+}$, but it decreased for $\mathrm{MB}^{+}$. This observation was explained by the sizes of the photosensitizers if the photosensitizer is small and buried in the cavity of the macrocycle that may cause the quenching of the generated singlet oxygen in the media. Moreover, they concluded that for photocleavage of the proteins to take place efficiently, some part of the inclusion complex of the photosensitizer should be available to interact with proteins. ${ }^{[38]}$

\subsection{Assembly of Cucurbiturils with Conjugated Oligomers and Polymers}

$\pi$-Conjugated oligomers and polymers have many potential applications including light emitting diodes, photovoltaics, solid state lighting, organic lasers as well as in the biomedical applications including actuators, sensors, cell imaging and labelling. ${ }^{[2-6]}$ The optical properties of these materials may need some tailoring to be fit for certain applications.

Although assembly of these materials with CBs could result in nanostructured materials with unprecedented properties, in the literature there are not many examples. The presence of CBs could improve the chemical, thermal and photostabilities of the conjugated materials as well as their solubilities in aqueous media.

The effect of CBs could be used in several ways; part of the conjugated backbone could be threaded with CBs either during the polymerization or post-threading could be done on the preformed conjugated backbone.

The first example of a conjugated rotaxane using the macrocycle CB was reported by Anderson et al. ${ }^{[39]}$ They have synthesized a CB7-encapsulated oligoaniline rotaxane using aqueous reductive amination and the effect of $\mathrm{CB}$ on the electronic properties of the oligoaniline. It was noted that $\mathrm{CB} 7$ binds strongly to the radical cation oligoaniline intermediates and the thermodynamic and kinetic stability of the radical cation increased by CB7-encapsulation; for instance, a $570 \mathrm{mV}$ reduction was observed in the first oxidation potential of the $\pi$-system which corresponds to an increase of the comproportionation constant by a factor of 109 . They expect that the threading of polyaniline by $\mathrm{CB}$ will affect significantly its electronic properties. ${ }^{[39]}$

Later on, Farcas et al reported the synthesis of CB7-based conjugated polyrotaxane which was synthesized through Suzuki Coupling between 9,9-dioctylfluorene-2,7-diboronic acid bis(1,3-propanediol) ester and an inclusion complex of 5,5'-dibromo-2,2'-bithiophene with CB7. ${ }^{[40]}$ The authors claimed to have $33 \%$ threading efficiency and the resulting polyrotaxane is soluble in DMSO but not in water because of the hydrophobic nature of the polymer backbone and side chains. It was noted that the fluorescence quantum yield of polyrotaxane was lower than that of its conjugated polymer counterpart but the fluorescence lifetime observed to be longer. They have also investigated the morphology of the polyrotaxane using atomic force microscopy and observed that polyrotaxanes self-assembled into extended ribbons whereas its polymer counterpart arranged into short C-shaped structures. $^{[40]}$

Our group has recently reported the synthesis and optical properties of the CB7-threaded conjugated polyelectrolytes. We observed, contrary to the previous report, a significant increase in the fluorescence quantum yield of the conjugated polyrotaxane compared to its polymer counterpart. Moreover, these polyrotaxanes soluble well in water and DMSO. ${ }^{[41]}$ The following conjugated polyrotaxanes and the conjugated polymers by Suzuki Coupling reactions were synthesized: Poly (9,9'-bis(6“-(N,N,N-trimethylammonium)-hexyl)fluorene-altco-thiophenelene) $\mathbf{P 1}$ and poly(9, 9'-bis(6"-(N,N,N trimethylammonium)propyl)fluorenealt-co-thiophenelene) $\mathbf{P 2}$ and their CB7-based polyrotaxane counterparts, P1CB7 and P2CB7. Threading efficiencies were estimated to be $50 \%$ and $15 \%$ for P1CB7 and P2CB7, respectively. Fluorescence quantum yields of $\mathbf{P 1}$ and $\mathbf{P 2}$ which are 0.11 and 0.35 have increased to 0.46 and 0.55 for P1CB7 ( $>4$ fold) and P2CB7, respectively and fluorescence lifetimes of the polyrotaxanes both in the solution and the solid state were observed to be longer than their polymers. Threading of the polymers by CB7 also increased their thermal stabilities. Light emitting diodes from P1 and its CB7-based polyrotaxane counterpart P1CB7 were constructed. Polyrotaxane-based diode showed lower turn-on voltages with high electroluminescence color purity due to balanced charge injection in P1CB7 as compared to the P1 polymer.

We observed that although isolating some part of the conjugated backbone could increase the solid state fluorescence quantum yield of the conjugated polymer and the emission quenching could be minimized compared to its polymer counterpart but still it requires some improvements. ${ }^{[42]}$ To achieve this, we have embedded them in organic crystalline matrices such as trehalose and sucrose by co-crystallization. As a result, we obtained a highly efficient color-converting solids suitable for solid-state lighting with high fluorescence quantum efficiency $(>50 \%)$. A proof-of-principle lightemitting diode is constructed by employing their powders as color converters. ${ }^{\text {[2] }}$ 
As it is mentioned earlier, conjugated polyrotaxanes or polypseudorotaxanes could also be prepared by threading the macrocycles onto the pre-synthesized polymers. ${ }^{[43]}$ Based on this idea, recently conjugated polypseudorotaxanes have been synthesized through the threading of cucurbiturils onto the cationic poly(pyridyl vinylene), PPyV. Among CB homologues, CB7 has been found to have the most suitable size that the pyridyl units could fit into and interact efficiently with cations through ion-dipole interaction. On the other hand, CB5 is too small to thread the backbone and CB8 provides loose fit that causes less efficient binding. CB6 is on the borderline as it can thread the backbone but the process observed to be too slow. Threading efficiency estimated as around $3 \%$ which is quite low and reason might be due the strong binding constant of pyridinium ion with $\mathrm{CB} 7$ as it binds with the terminal pyridinium groups on the chain and behave like stoppers which does not allow the polymer chain to be further threaded. Although the threading efficiency is quite low, still an increase in the fluorescence quantum yield was observed as well as an increase in the water solubility. Figure 6 shows a cartoon presentation of the structure of PpyV-CBn-Polypseudorotaxane. The authors also demonstrated the sensing properties of these conjugated polypseudorotaxane with electron rich analytes by taking advantage of the electron poor properties of the polymer backbone. ${ }^{[43]}$

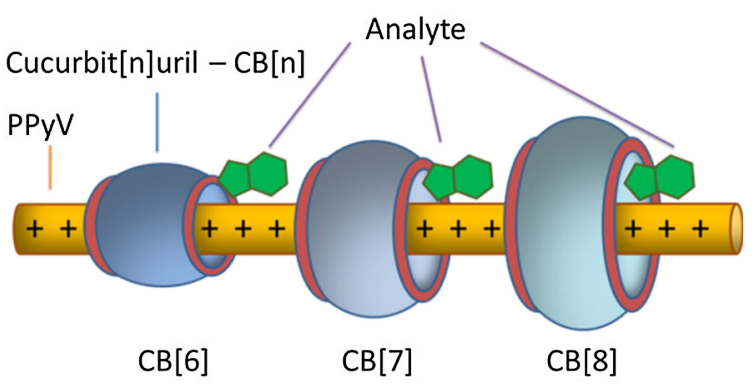

PPyV-CB[n] Poly-pseudo-rotaxane

Figure 6. Cartoon presentation of the structure of PpyV-CBn Polypseudorotaxane. (Reprinted with permission from ref. 27. Copyright 2016 American Chemical Society.)

Do the backbone of the conjugated oligomers or the polymers should be threaded or encapsulated by CBs in order to influence the physical, chemical and thermal properties of these materials? In the following section, we will present examples to find answers to this question.

For instance, in our group we investigated the effects of $\mathrm{CBn}$ on the dissolution and the photophysical properties of nonionic conjugated polymers in water. ${ }^{[44]}$ For this purpose, poly [9, 9-bis $\{6(\mathrm{~N}, \mathrm{~N}$-dimethylamino)hexyl $\}$ fluoreneco-2,5thienylene (PFT) was synthesized and its optical properties have been investigated in the presence and the absence of $\mathrm{CB}$ homologues. We have observed that this nonionic conjugated polymer can be made soluble in water through an inclusion complex formation with CB8. This complex emits green and is highly fluorescent with fluorescence quantum yield of $35 \%$. It was also observed that the morphology of the polymer PFT was affected by the presence of CB8. Thus CB8-assisted selfassembly of polymer chains leads to vesicles formation; these structures were characterized by DLS, AFM, SEM, TEM and fluorescence optical microscopy. Although we have tried other CB homologues (CB6 and CB7), no changes have been observed in their solubility behavior and photophysical properties. ${ }^{[44]}$

In another study, the effect of the CBs have been demonstrated in an image-guided controlled release drug delivery by using a conjugated oligomer-based nanoparticles. ${ }^{[4]}$ Conjugated oligomer was synthesized through Heck coupling of divinylfluorene and dibromobenzothiodiazole monomers and has an amphiphilic nature due to the presence of tertiary alkyl amine residues as pendant of the fluorene monomer. This oligomer self-assembled in water to form particles. These red emitting water-dispersible nanoparticles could carry camptothecin, an anticancer drug with high loading efficiency. MTT cell viability studies performed with breast cancer cell lines showed that half maximal inhibitory concentration (IC50) values of nanoparticles for MCF7 and MDA-MB-231 were $44.7 \mu \mathrm{M}$ and $24.8 \mu \mathrm{M}$, respectively which was found to be a little bit high. Therefore, amine groups were disguised by capping with CB7 to decrease the cytotoxicity and increase the stability of nanoparticles. Drug release studies showed that drugs were released at low $\mathrm{pH}$ (at 5.0) faster than physiological $\mathrm{pH}$ (7.4) confirming the $\mathrm{pH}$ responsive nature of the nanoparticles. On the other hand, CB7-capped drug-loaded nanoparticles regulated the release rate by providing slower release at $\mathrm{pH} 7.4$ than the nanoparticles in the absence of CB7 s. IC50 values for camptothecin in the presence of nanoparticles with or without CB7 were significantly reduced in MCF7 and MDAMB-231 cells..$^{[45]}$

Cationic conjugated polyelectrolytes can be used in photodynamic antibacterial chemotherapy in which cationic ammonium groups interact with the negatively charge bacterial cell membranes and the photoactive backbone of the polymers acts as a photosensitizer that can be irradiated by light to generate the reactive oxygen species for photodynamic effect.

Wang et al designed a supramolecular antibiotic switch in which CB7 and amantadine (AD) are used to turn-on or turnoff the biocidal activity of cationic poly(phenylene vinylene) derivatives (PPV) ${ }^{[47]}$ Antibacterial activity of the cationic PPV was regulated by complexation and de-complexation of $\mathrm{CB} 7$ with its ammonium groups. PPV/CB7 complex reduce the biocidal activity of PPV by encapsulating quaternary ammonium groups (switch off). Upon adding AD which has a higher affinity toward CB7 than the ammonium groups, the more stable CB7/AD complex forms and releases PPV through competitive replacement. Thus, the antibacterial activity of $\mathrm{PPV}$ is recovered (switch on). ${ }^{[46]}$

Some modification was done to improve the effectiveness of this supramolecular antibiotic switch using a conjugated 
polyelectrolyte, cationic poly(fluorene-co-phenylene) derivative (PFP), CB7 and a nonionic surfactant Triton X-100 (polyoxyethylene octyl phenyl ether). ${ }^{[4]}$ PFB showed some different behavior than the cationic PPV used in the previous study. For instance, both PFB and its supramolecular complex with CB7 exhibited high antimicrobial effect against the bacteria. So that the presence of CB7 has not affected much the availability of the cationic ammonium groups and as a result, no distinctive switching properties are observed. The reason was explained by the uncontrolled aggregate formation of PFP chains in water. To this end, Triton X-100 was employed to control the aggregation state before supramolecular assembly/disassembly of PFP with CB7 occurred. The resulting complex acts as an efficient supramolecular antibiotic switch for reversibly controlling the antibacterial activity both in the dark and under white light irradiation. Figure 7 shows the antibacterial process of the antibiotic switch with or without pretreatment with Triton X100 .

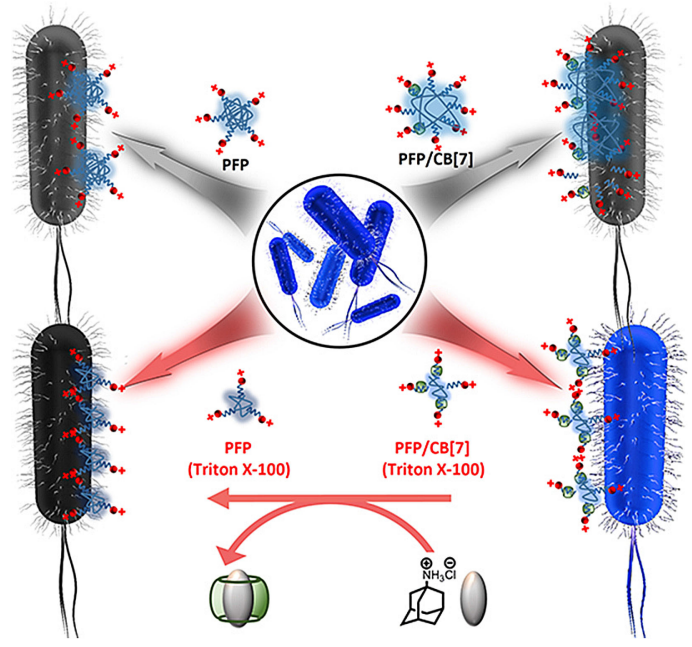

Figure 7. Schematic presentation of the antibacterial process of the antibiotic switch with or without pretreatment with Triton X-100. (Reprinted with permission from ref. 47. Copyright 2017 American Chemical Society.)

In another study, the supramolecular complex of cationic poly(fluorene-co-phenylene) derivative $\left(\mathrm{PFPNMe}^{3+}\right.$ ) with $\mathrm{CB} 7$ has been utilized in situ pathogen detection and discrimination of multiple pathogens by taking advantage of optical spectral changes of the complex before and after disassembly on the pathogen surfaces. ${ }^{[48]}$ Through SEM, DLS, and zeta potential measurements, it was shown that the PFP-NMe ${ }^{3+} / \mathrm{CB}$ [7] complex has different degree of electrostatic and hydrophobic interactions with various pathogens before and after disassemble. This supramolecular complex acts as an optical sensor in the detection of the Gram-negative bacteria (E. coli, P. aeruginosa), Gram-positive bacteria (B. subtilis, S. aureus, E. faecalis), and the fungi (C. albicans, S. cerecisiae) and can also discriminate different strains of the same species. Moreover, it was reported that a blend sample of three pathogens could also be identified by this sensor. ${ }^{[48]}$

\section{Summary and Outlook}

In this review, we discussed CB-containing supramolecular structures based on $\pi$-conjugated, optically active compounds including small fluorescent dyes, porphyrin derivatives, conjugated oligomers and polymers. Depending on the sizes and chemical nature of the guest chromophores, $\mathrm{CBn}$ homologues either fully or partially encapsulate them to form inclusion complexes. Upon complexation, the optical properties of these chromophores were significantly altered; for instance, their fluorescence quantum yields increased while extending their lifetimes and enhancing their photostability. Moreover, through complexation the solubility of the chromophores increased and the aggregate formation was minimized. The use of these CB-containing supramolecular structures have also been demonstrated in the biomedical and photonic applications. For instance, CB-threaded conjugated polymers have been used as an efficient color converter and utilized in the construction of light emitting diodes. Porphyrin and conjugated polyelectrolyte-based chromophores have been employed as CB-regulated photosensitizers in photodynamic antibacterial therapy and image guided targeted delivery.

Although especially the assembly of conjugated oligomers and polymers with $\mathrm{CBs}$ could result in nanostructured materials with unprecedented properties for various important applications, there are limited number of examples in the literature.

\section{Acknowledgements}

We thank TÜBİTAK for funding (KBAG $114 Z 195$ and KBAG 215Z035).

\section{References}

[1] K. Nakano, K. Tajima, Adv. Mater. 2017, 29, 1603269, DOI: 10.1002/adma.201603269.

[2] A. Facchetti, Chem. Mater. 2011, 23, 733.

[3] L. Feng, C. Zhu, H. Yuan, L. Liu, F. Lv, S. Wang, Chem. Soc. Rev. 2013, 42, 6620.

[4] A. C. Grimsdale, K. L. Chan, R. E. Martin, P. G. Jokisz, A. B. Holmes, Chem. Rev. 2009, 109, 897.

[5] S. E. Root, S. Savagatrup, A. D. Printz, D. Rodriquez, D. J. Lipomi, Chem. Rev. 2017, 117, 6467.

[6] F. J. M. Hoeben, P. Jonkheijm, E. W. Meijer, A. P. H. J. Schenning, Chem. Rev. 2005, 105, $1491-1546$.

[7] R. Behrend, E. Meyer, F. Rusche, Liebigs Ann. Chem. 1905, 339, $1-37$.

[8] a) W. A. Freeman, W. L. Mock, N. Y. Shih, J. Am. Chem. Soc. 1981, 103, 7367-7368; b) W. L. Mock, in Comprehensive 
Supramolecular Chemistry, ed. F. Vogtle, Pergamon Press, Oxford, 1996, vol. 2, pp. 477-493.

[9] a) J. Kim, I.-S. Jung, S.-Y. Kim, E. Lee, J.-K. Kang, S. Sakamoto, K. Yamaguchi, K. Kim, J. Am. Chem. Soc. 2000, 122, 540-541; b) A. Day, A. P. Arnold, R. J. Blanch, B. Snushall, J. Org. Chem. 2001, 66, 8094-8100.

[10] a) E. Masson, X. Ling, R. Joseph, L. Kyeremeh-Mensah, X. Lu, $R S C A d v .2012$, 2, 1213-1247; b) S. Gürbüz, M. Idris, D. Tuncel, Org. Biomol. Chem. 2015, 13, 330-347; c) K. I. Assaf, W. M. Nau, Chem. Soc. Rev. 2015, 44, 394-418; d) D. Shetty, J. K. Khedkar, K. M. Park, K. Kim, Chem. Soc. Rev. 2015, 44, 87478761; e) S. J. Barrow, S. Kasera, M. J. Rowland, J. Del Barrio, O. A. Scherman, Chem. Rev. 2015, 115, 12320-12406.

[11] a) Z. Li, S. K. M. Nalluri, J. F. Stoddart, Chem. Soc. Rev. 2017, 46, 2459-2478; b) J. Murray, K. Kim, T. Ogoshi, W. Yao, B. C. Gibb, Chem. Soc. Rev. 2017, 46, 2479-2496;

[12] a) D. Tuncel, O. Unal, M. Artar, Isr. J. Chem. 2011, 51, 525-532; b) D. Tuncel, H. B. Tiftik, B. Salih, Chem. Commun. J. Mater. Chem. 2006, 16, 3291-329; c) D. Tuncel, J. H. G. Steinke, Macromolecules 2004, 37, 288.

[13] a) W. M. Nau, A. Hennig, A. L. Koner, Fluorescence of Supermolecules, Polymers, and Nanosystems, Springer, 2008, pp. 185211; b) A. L. Koner, W. M. Nau, Supramol.Chem. 2007, 19, 5566.

[14] R. N. Dsouza, U. Pischel, W. M. Nau, Chem. Rev. 2011, 111, 7941-7980.

[15] K. I. Assaf, W. M. Nau, Supramol. Chem. 2014, 26, 657-669.

[16] A. C. Bhasikuttan, H. Pal, J. Mohanty, Chem. Commun. 2011, 47, 9959-9971.

[17] A. Praetorius, D. M. Bailey, T. Schwarzlose, W. M. Nau, Org. Lett. 2008, 10, 4089-4092.

[18] S. S. Thakare, G. Chakraborty, P. Krishnakumar, A. K. Ray, D. K. Maity, H. Pal, N. Sekar, J. Phys. Chem. B 2016, 120, $11266-11278$.

[19] J. Mohanty, K. Jagtap, A. K. Ray, W. M. Nau, H. Pal, Chem. Phys. Chem. 2010, 11, 3333-3338.

[20] M. Sayed, F. Biedermann, V. D. Uzunova, K. I. Assaf, A. C. Bhasikuttan, H. Pal, W. M. Nau, J. Mohanty, Chem. Eur. J. 2015, 21, 691-696.

[21] F. Biedermann, E. Elmalem, I. Ghosh, W. M. Nau, O. A. Scherman, Angew. Chem. Int. Ed. 2012, 51, 7739-7743.

[22] X. Song, F. Liu, S. Sun, J. Wang, J. Cui, X. Peng, RSC Adv. 2014, 4, 9326-9329.

[23] M. E. Aliaga, L. Garcia-Rio, M. Pessego, R. Montecinos, D. Fuentealba, I. Uribe, M. Martin-Pastor, O. Garcia-Beltran, New J. Chem. 2015, 39, 3084-3092.

[24] C. P. Carvalho, R. Ferreira, J. P. Da Silva, U. Pischel, Supramol. Chem. 2013, 25, 92-100.

[25] X.-Q. Wang, Q. Lei, J.-Y. Zhu, W.-J. Wang, Q. Cheng, F. Gao, Y.-X. Sun, X.-Z. Zhang, ACS Appl. Mater. Interfaces 2016, 8, $22892-22899$.

[26] a) I. Beletskaya, V. S. Tyurin, A. Y. Tsivadze, R. Guilard C. Stern, Chem. Rev. 2009, 109, 1659-1713; b) C. M. Drain, A.
Varotto, I. Radivojevic, Chem. Rev. 2009, 109, 1630-1658; c) N. Aratani, D. Kim, A. Osuka, Acc. Chem. Res. 2009, 42, 19221934; d) T. S. Balaban, Acc. Chem. Res. 2005, 38, 612-623.

[27] B. Girek, W. Sliwa, J. Incl. Phenom. Macrocycl. Chem. 2015, 81, 35-48.

[28] D. Tuncel, N. Cindır, U. Koldemir, J. Incl. Phenom. Macrocycl. Chem. 2006, 55, 373-380.

[29] S. Liu, A. D. Shukla, S. Gadde, B. D. Wagner, A. E. Kaifer, L. Isaacs, Angew. Chem. Int. Ed. 2008, 47, 2657-2660.

[30] J. Mohanty, A. C. Bhasikuttan, S. D. Choudhury, H. Pal, J. Phys. Chem. B 2008, 112, 10782-10785.

[31] N. Barooah, A. C. Bhasikuttan, V. Sudarsan, S. D. Choudhury, H. Pal, J. Mohanty, Chem. Commun. 2011, 47, 9182-9184.

[32] X. Xiao, W. Li, J. Jiang, Inorg. Chem. Commun. 2013, 35, 156159.

[33] W. Lei, G. Jiang, Q. Zhou, Y. Hou, B. Zhang, X. Cheng, X. Wang, Chem. Phys. Chem. 2013, 14, 1003-1008.

[34] M. Fathalla, N. L. Strutt, J. C. Barnes, C. L. Stern, C. Ke, J. F. Stoddart, Eur. J. Org. Chem. 2014, 2873-2877.

[35] K. Liu, Y. Liu, Y. Yao, H. Yuan, S. Wang, Z. Wang, X. Zhang, Angew. Chem., Int. Ed., 2013, 52, $8285-8289$.

[36] L. Chen, H. Bai, J-F. Xu, S. Wang, X. Zhang, ACS Appl. Mater. Interfaces 2017, 9, 13950-13957.

[37] W. Lei, G. Jiang, Q. Zhou, B. Zhang, X. Wang, Phys. Chem. Chem. Phys. 2010, 12, 13255-13260.

[38] J. Caceres, J. Robinson-Duggon, A. Tapia, C. Paiva, M. Gomez, C. Bohne, D. Fuenteal, Phys. Chem. Chem. Phys. 2017, 19, 2574.

[39] R. Eelkema, K. Maeda, B. Odell, H. L. Anderson, J. Am. Chem. Soc. 2007, 129, 12384.

[40] A. Farcas, P.-H. Aubert, J. Mohanty, A. I. Lazar, S. Cantin, W. M. Nau, European Polymer Journal 2015, 62, 124-129.

[41] M. Idris, M. Bazzar, B. Guzelturk, H. V Demir, D. Tuncel, RSC Adv. 2016, 6, 98109.

[42] T. Erdem, M. Idris, H. V. Demir, D. Tuncel, Macromol. Mater. Eng. 2017, 1700290.

[43] N. Willis-Fox, C. Belger, J. F. Fennell, R. C. Evans, T. M. Swager, Chem. Mater. 2016, 28, 2685.

[44] D. Tuncel, M. Artar, S. B. Hanay, J. Polym. Sci. Part A Polym. Chem. 2010, 48, 4894.

[45] J. Pennakalathil, E. Jahja, E. Sıla Özdemir, Ö. Konu, D. Tuncel, Biomacromolecules 2014, 15, 3366-3374.

[46] H. Bai, H. Yuan, C. Nie, B. Wang, F. Lv, L. Liu, S. Wang, Angew. Chem., Int. Ed. 2015, 54, 13208-13213.

[47] H. Bai, H. Zhang, R. Hu, H. Chen, F. Lv, L. Liu, S. Wang, Langmuir 2017, 33, 1116-1120.

[48] H. Bai, H. Chen, R. Hu, M. Li, F. Lv, L. Liu, S. Wang, ACS Appl. Mater. Interfaces 2016, 8, 31550-31557.

Received: September 30, 2017

Accepted: October 29, 2017

Published online on November 14, 2017 